\title{
EPSDT Care and Emergency Department Visits for Non-Urgent Care: Hispanic vs. Non-Hispanic Children in a Border Community
}

\section{Richard J Butler ${ }^{1 *}$ and William G Johnson ${ }^{2}$}

${ }^{1}$ Department of Economics, Brigham Young University, Provo, Utah, USA

${ }^{2}$ Department of Medical Bioinformatics, Arizona State University, USA

*Corresponding author:Richard J Butler, Martha Jane Knowlton Coray Professor, Department of Economics, Brigham Young University, Provo, Utah, USA, Tel: 801-422-1372; E-mail: richard_butler@byu.edu

Received date: March 01, 2016; Accepted date: March 15, 2016; Published date: March 22, 2016

Copyright: (c) 2016 Butler RJ, et al. This is an open-access article distributed under the terms of the Creative Commons Attribution License, which permits unrestricted use, distribution, and reproduction in any medium, provided the original author and source are credited.

\begin{abstract}
Objectives:We examine the use of emergency departments for non - urgent care, comparing children who received recommended EPSDT services to those who do not, analyzing differences between Hispanic and Non Hispanic children, controlling for differences in their characteristics.

Methods: A retrospective, longitudinal study of a cohort of approximately 6,000 children during the years from 1999 - 2009 using data from hospital and emergency department (ED) claims, paediatric office visits and claims data from Medicaid (AHCCCS) and SCHIP. Three different versions of an econometric model are presented. The estimates also include the effects of health insurance coverage and the lack thereof by evaluating the records of all children age 0 - 19 in Yuma County who met any of the following criteria: insured by Medicaid or SCHIP at any time; or used the emergency department (ED) or any other participating provider during the ten years; or were immunized. We test if the provision of recommended EPSDT services by age two, affected the subsequent number of nonurgent care visits to hospital's ED.
\end{abstract}

Results: Children without continuous health insurance coverage are twice as likely as continuously insured children to use and ED for non-urgent care. Controlling for health insurance coverage and demographics, the receipt of recommended EPSDT care by age two significantly reduces non-urgent care visits to the ED for Hispanic children. In some instances the likelihood of such visits is cut in half.

Conclusion: The results suggest that the provision of EPSDT services to the growing number of Hispanic children can yield significant benefits.

Keywords: EPSDT care; Hispanic health; Border health services; Emergency department

\section{Introduction}

EPSDT services are claimed to reduce hospitalizations and emergency department (ED) visits for conditions for which primary care is more cost-effective [1,2]. Empirical tests of the claim are, however, rare and often restricted to small samples because the benefits occur over long periods of time. This research estimates the effect of EPSDT services on subsequent ED visits for non-urgent care (NED) visits among a cohort of approximately six-thousand children between 1999 - 2009. The results include the differential effects of EPSDT services between Hispanic $(\mathrm{H})$ and Non - Hispanic White (NHW) children. The setting is Yuma county, a 5,000 square mile, semi - rural county, on the U.S, Mexican border.

\section{Background}

EPSDT services include blood pressure monitoring, blood and urine tests, developmental and behavioural assessments, hearing and sight screenings, immunizations, lead screening, medical histories, new born screening, regular physical examinations, treatment for certain chronic conditions and guidance for injury prevention [3-5]. Guideline consistent EPSDT care has been shown to reduce hospitalizations, regardless of race, poverty, or health status [6].

Hispanic children may benefit more from EPSDT visits than NHW children because they are more likely to be uninsured $[7,8]$, have fewer physician visits; longer intervals between visits, and poorer health status [7]. Despite the potential benefits, Hispanic children receive fewer EPSDT visits than NHW children [9-11]. We estimate the effect of EPSDT services on ED visits for NED visits for a large sample of Arizona children over a decade and analyze the differences in the receipt of EPSDT services and outcomes between Hispanic and NHW children.

\section{Data}

The sample was drawn from Arizona Health Query, a state wide warehouse of health care claims. Inpatient and ED data are contributed by Yuma Regional Medical Center (YRMC), the only hospital and ED in Yuma County. The nearest alternative hospital is approximately 180 miles away so it is likely that the data include almost all hospitalizations and ED visits for this patient population. During the study period, the ED provided more than 45,000 visits annually. The other data sources include Yuma's two largest paediatric practices, its 
Citation: Butler RJ, Johnson WG (2016) EPSDT Care and Emergency Department Visits for Non-Urgent Care: Hispanic vs. Non-Hispanic Children in a Border Community. Health Econ Outcome Res Open Access 2: 112. DOI: 10.4172/2471-268X.1000112

Page 2 of 7

largest OB / GYN practice and Medicaid (AHCCCS) and SCHIP (Kids Care).

The encounter data include health insurance coverage by insurer (all payers), diagnostic information (ICD9), procedures (CPT codes), times of treatment and demographics. Approximately $70 \%$ of Yuma children were covered by Medicaid / SCHIP during the study years. The sample includes 4,525 $\mathrm{H}$ children and 1,640 NHW children. The large number of Hispanic children reflects the ethnic composition of the County which is on the border with Sonora, Mexico. Children are omitted from the data if they satisfy all of the following conditions for all years 1999 - 2009: (1) not insured by Medicaid or SCHIP at any time; and (2) did not use the ED or any other participating provider during the ten years; and (3) not immunized (immunization is required for public school enrolment).
Using children as their own controls eliminates many potential biases. If, for example, new children were added at different points in time, their family background and other unobservable characteristics might be systematically different from those of the children in the original cohort, confounding the treatment effect.

There are three mutually exclusive groups of children, namely: those whose EPSDT visits ended by age 2, 3 or 4, respectively. The age 2 groups is the reference against which the experiences of the other age groups are compared (many screening recommendations are for children by age 2 [12]). Table 1 shows that the distributions of EPSDT visit for $\mathrm{H}$ and NHW children are similar although the numbers of visits are different. The modal number of visits by age 2 is zero; the median is 3 - 4 visits for both groups. One-fourth of the children in each group have no visits and another one-fourth have seven or more.

\begin{tabular}{|c|c|c|}
\hline Number of EPSDT visits by Age 2 & Hispanics & Non - Hispanic White \\
\hline 0 & 2342 & 880 \\
\hline 1 & 963 & 244 \\
\hline 2 & 963 & 228 \\
\hline 3 & 910 & 233 \\
\hline 4 & 880 & 269 \\
\hline 5 & 864 & 277 \\
\hline 6 & 954 & 298 \\
\hline 7 & 893 & 329 \\
\hline 8 & 788 & 283 \\
\hline 9 & 511 & 257 \\
\hline 10 & 298 & 154 \\
\hline 11 & 135 & 28 \\
\hline 12 & 61 & 11 \\
\hline 13 & 11 & 0 \\
\hline 14 & 6 & 0 \\
\hline 15 & 3 & 0 \\
\hline 17 & 1 & 0 \\
\hline 18 & 1 & 4 \\
\hline 21 & 0 & 1 \\
\hline 25 & 1 & 0 \\
\hline 26 & 1 & 0 \\
\hline
\end{tabular}

Table 1: EPSDT visits by ethnicity.

A child may be observed more than once; a child with 7 years of data would, for example, contribute 7 child-year observations. There are 10,586 child-year observations for Hispanics and 3,496 child-year observations for NHWs (Table 2). Although similar in terms of age and gender, NHWs have more EPSDT visits than Hispanics (4.18 versus, 3.96) and fewer NED visits per year (7.75\% versus $9.07 \%$ per child-year). The ethnic differences in NED visits may partly reflect differences in location. NHW children live closer to the YRMC ED in the city of Yuma, while $\mathrm{H}$ children more often live in smaller outlying towns. 
Citation: Butler RJ, Johnson WG (2016) EPSDT Care and Emergency Department Visits for Non-Urgent Care: Hispanic vs. Non-Hispanic Children in a Border Community. Health Econ Outcome Res Open Access 2: 112. DOI: 10.4172/2471-268X.1000112

Page 3 of 7

\begin{tabular}{|l|l|l|l|l|}
\hline \multicolumn{2}{|l|}{} & \multicolumn{2}{l|}{ Hispanics } & \multicolumn{2}{l|}{ Non - Hispanic NHWs } \\
\hline & means & standard dev. & means & standard dev. \\
\hline Nonurgent prob, per child year & 0.09 & 0.287 & 0.078 & 0.267 \\
EPSDT visits (by 2nd year) & 3.96 & 3.272 & 4.18 & 3.441 \\
after"EPSDT visits & 1.69 & 2.863 & 1.61 & 2.892 \\
after & 0.44 & 0.495 & 0.40 & 0.489 \\
male & 0.54 & 0.498 & 0.499 \\
health condition present & 0.15 & 0.356 & 0.339 \\
private insurance coverage & 0.23 & 0.421 & 0.13 & 0.484 \\
Medicaid / SCHIP coverage & 0.73 & 0.442 & 0.38 & 0.494 \\
gaps in insurance coverage & 0.03 & 0.167 & 0.43 & 0.169 \\
z85349 & 0.17 & 0.373 & 0.03 & 0.113 \\
z85350 & 0.13 & 0.335 & 0.03 & 0.167 \\
z85356 & 0.01 & 0.109 & 0.02 & 0.149 \\
z85364 & 0.53 & 0.498 & 0.56 & 0.496 \\
z85365 & 0.09 & 0.287 & 0.26 & 0.436 \\
z85366 & 0.02 & 0.139 & 0.02 & 0.142 \\
z85367 & 0.02 & 0.137 & 0.07 & 0.258 \\
\hline Sample size, number of children & 4,525 & 1,640 & \\
\hline Sample size (children years) & 10,586 & 3,496 & \\
\hline
\end{tabular}

Table 2: Sample means for non - urgent emergency department (NED) visits per child year data for 1999 - 2009, using all non - missing observations.

\section{Methods}

\section{Definitions of variables}

Age: Age in years on December 31st of each calendar year. Dummy variables are used for each year of age from $0-9$ with age $10+$ as reference. After is a dummy for a child after two years of age.

Male: A dummy variable equal to 1 if the child is male, else 0.

Hispanic (for Race / Ethnicity): Race / ethnicity are defined as Hispanic (regardless of race) and NHW, based on providers' records. The data are stratified by ethnicity to capture ethnicity effects that have been found to be important in previous research $[7,8]$.

Transportation / geography: Yuma County encompasses 5,000 square miles with one medium sized city, two small towns and widely scattered clusters of residences in the desert. There was no public transportation in Yuma County during the study period. We include dummy variables for zip codes as controls for transportation / geography.

Insurance: We capture whether or not a child is insured (coverage status), and, if insured, the type of insurance coverage (type status).

Gaps in insurance coverage is a dummy variable equal to 1 if a child's care in a year was not insured or included insured and uninsured episodes. The comparison group consists of children who were continuously insured and children whose insurance status is unknown. The inclusion of observations with unknown insurance had no effect on any of the results reported here.
Type status is represented by: Medicaid / SCHIP and Private insurance. Medicaid is a 1,0 dummy variable equal to 1 if the child had a visit during the year that was paid by Medicaid / SCHIP, Private insurance is a dummy variable equal to one if the child had at least 1 visit in a year paid by private insurance. Health conditions is a 1,0 dummy equal to 1 if episodes of care for any of the following medical conditions, (ICD - 9 codes) occurred two or more times during a year: asthma, cardiac problems, congenital anomalies, dental caries, endocrine disorders including diabetes, epilepsy, mental health or development disability, malignant cancer, nutritional disorders (including obesity and failure to thrive), otitis media, paralysis, pregnancy, pulmonary tuberculosis and urinary tract infections.

EPSDT visit is a count variable for the number of EPSDT visits delivered in a year based on ICD, CPT, and / or HCPS diagnosis and procedure codes.

\section{Outcome variable}

NED Visits is a 1,0 dummy equal to 1 if a child had a NED visit in a year. ED visits accompanied by hospitalization on the same day are considered urgent visits. We follow two studies and use CPT codes to further identify urgent visits $[13,14]$. Visits that required an imaging study (e.g., CT scan, MRI, X - ray) or procedures not commonly performed in outpatient offices were considered urgent.

\section{Multivariate Estimates of EPSDT Effects}

Our 'difference - in - difference' estimate compares NED visits for each child before and after 2 years of age. This is the 'difference' in the 'difference - in - difference' estimator. The second difference compares 
Citation: Butler RJ, Johnson WG (2016) EPSDT Care and Emergency Department Visits for Non-Urgent Care: Hispanic vs. Non-Hispanic Children in a Border Community. Health Econ Outcome Res Open Access 2: 112. DOI: 10.4172/2471-268X.1000112

Page 4 of 7

the before and after age 2 NED visits for children receiving EPSDT visits (the treatment group) to before and after NED visits for children with no EPSDT visits. The estimator of the effect of EPSDT visits on NED visits becomes:

(NED visits after 2 - NED visits before 2) EPSDT- (NED visits after 2 - NED visits before 2) no EPSDT

Where the "EPSDT" and "no EPSDT" superscripts indicate children with the recommended number of EPSDT treatments by age 2, and those with less than that number. Three different specifications of equation (1) are embedded in models with additional control variables, namely: a random effects model; a non - linear "cliff" model and a non-parametric age model.

\section{Random effects model}

The longitudinal data permit difference - in - difference estimates with the inclusion of a separate response for each child. We assume that the separate responses are uncorrelated with the independent variables and estimate a random effects model (using proc genmod in SAS). The model is used to estimate the effect of receiving the recommended number of EPSDT visits by age 2 on the likelihood of an NED visit in a given year after age two.

The difference - in - difference estimate of the EPSDT treatment (Equation 1) is the interaction of the EPSDT dummy variable and the, EPSDT $^{*}$ after variable. The coefficient on EPSDT ${ }^{\star}$ after estimates the effect of guideline consistent EPSDT visits by age two on the subsequent annual likelihood of NED visits, controlling for age, distance to the ED, gender, insurance effects and health conditions.

\section{Non - linear “Cliff” model}

If it takes several visits for the EPSDT services to be effective, the Random Effects model may impose a continuity that disguises the possibility that the effects of EPSDT visits act more like a cliff than a mountain slope. To address this possibility, we replicate the Random Effects model but compare children with $0-1$ EPSDT visits (the control), to children with 7 or more EPSDT visit by the age 2 (the treatment group).

\section{Non - parametric age model}

This specification replicates the analysis using individual age dummy variables instead of an "after" variable, allowing children's age to have a non - parametric (i.e., not con strained or pre - specified) effect on ED use.

\section{Random Effects Model Results}

There is no significant gender effect for NED visits in Table 3: Hispanic males visit the ED slightly more than Hispanic females, and NHW males slightly less than NHW females, but neither effect is statistically significant. Medicaid insurance coverage decreases NED visits among NHW children by 4.8 percentage points or approximately 60 percent $(0.048 / 0.078)$. Private insurance coverage is not a statistically significant influence on NHW children while Medicaid / SCHIP coverage is not a statistically significant influence on NED visits among $\mathrm{H}$ children. Private insurance coverage for $\mathrm{H}$ children, however, increases the likelihood of an NED visit by 2.7 percentage points, or nearly 30 percent $(0.027$ / 0.091). In this case, private insurance's effect in increasing the overall demand for care is outweighing the substitution effect where private doctor visits are substituted for NED visits (perhaps more off-hours emergency calls to doctors are being referred to the ED) (Table 3).

\begin{tabular}{|c|c|c|c|c|}
\hline & \multicolumn{2}{|l|}{ Hispanics } & \multicolumn{2}{|c|}{ Non - Hispanic NHWs } \\
\hline & coefficient & prob. signif. & coefficient & prob. signif. \\
\hline Intercept & -2.72 & $<0.001$ & -3.54 & $<0.001$ \\
\hline EPSDT visits (by 2nd year) & 0.01 & 0.430 & -0.02 & 0.381 \\
\hline after ${ }^{*}$ epsdt visits & -0.05 & 0.023 & 0.02 & 0.535 \\
\hline after & 0.48 & 0.001 & 0.28 & 0.260 \\
\hline male & 0.10 & 0.162 & -0.17 & 0.205 \\
\hline health condition present & -0.01 & 0.924 & 0.02 & 0.921 \\
\hline Medicaid insurance & -0.26 & 0.121 & -0.66 & 0.001 \\
\hline Private insurance coverage & 0.27 & 0.090 & 0.11 & 0.532 \\
\hline gaps in insurance coverage & 1.29 & $<0.001$ & 1.24 & $<0.001$ \\
\hline Diff. - in - diff. marginal effect (probability significance) & \multicolumn{2}{|c|}{$-0.0033(0.007)$} & \multicolumn{2}{|c|}{$0.0011(0.319)$} \\
\hline Log likelihood & \multicolumn{2}{|l|}{-3002.3787} & \multicolumn{2}{|l|}{-893.7924} \\
\hline Sample size (children years) & \multicolumn{2}{|l|}{10,586} & \multicolumn{2}{|l|}{3,496} \\
\hline Test: insurance effects & \multicolumn{2}{|l|}{$<0.001$} & \multicolumn{2}{|l|}{$<0.001$} \\
\hline Test year fixed effects & \multicolumn{2}{|l|}{$<0.001$} & \multicolumn{2}{|l|}{$<0.001$} \\
\hline Test: zipcode effects & \multicolumn{2}{|l|}{0.021} & \multicolumn{2}{|l|}{0.259} \\
\hline Test: Hispanic / NHW equality & \multicolumn{4}{|l|}{$<0.001$} \\
\hline
\end{tabular}

Table 3: Non - urgent (NED) visits per child-year logistic regressions. All probability significance levels have been adjusted for clustering at the individual level, and adjusted for heteroskedasticity using Robust Standard Errors. The difference - in - difference marginal effect in the last line is calculated using the Ai and Norton [15] corrections. Year fixed effects and Zip Code fixed effects are included in these models, but not reported in the table. 
Citation: Butler RJ, Johnson WG (2016) EPSDT Care and Emergency Department Visits for Non-Urgent Care: Hispanic vs. Non-Hispanic Children in a Border Community. Health Econ Outcome Res Open Access 2: 112. DOI: 10.4172/2471-268X.1000112

Page 5 of 7

Gaps in insurance coverage have the largest effect on the likelihood of NED visits for both $\mathrm{H}$ and NHW children. Children who were uninsured for all or part of a year were, all else equal, more than twice as likely as insured children to have a NED visit. Among Hispanic children, for example, the likelihood increases by 10.7 percentage points $\left(1.29^{*} 0.091^{*}(1-0.091)\right)$, more than doubling the 9.1 percent likelihood of a NED visit for a Hispanic child. The corresponding results for NHW children are a 0.09 percentage point increase also $\left(1.24^{\star} 0.078(1-0.078)\right)$,more than doubling the likelihood of using the NEO.

The estimated EPSDT ${ }^{*}$ after effect, the regression equivalent to the difference-in-difference estimator, is negative and statistically significant for $\mathrm{H}$ children (with a probability significance level of 2.3 percent - 023 - as indicated), but there is no significant effect for NHW children (with probability significance of only 0.535 ). The estimated interaction effect for Hispanics (after ${ }^{*}$ EPSDT visits coefficient $=$ -0.0516) in the left hand column of Table 3 indicates that guideline consistent EPSDT visits reduce the likelihood of an NED by about 0.5 percentage points for $\mathrm{H}$ children. However, as this interaction is embedded in a non-linear probability model, the actual difference-indifference effect has to account for the non-linearity between the EPSDT, after, and the EPSDT ${ }^{*}$ after coefficients, and their respective covariance's to make the appropriate inferences using the Ai and Norton correction [15]. The correction depends on all of the variables in the model (here we calculate the effect at the sample mean of the other variables) [15]. Employing this correction, the -0.5 percentage point effect becomes -0.33 , as indicated in the first row below the coefficient estimates (i.e., the -0.0033 value) The effect is both large and statistically significant $(<0.0001$ based on $\mathrm{Ai}$ and Norton adjustments [15]). Thus, in each year after the age of 2, Hispanic children are 3.6 percent less likely $(-0.0033$ / 0.0907) to have a NED visit if they received the recommended number of EPSDT visits by age two. That is, a $\mathrm{H}$ child with the recommended EPSDT visits is about a third less likely to have a NED visit each year, than a child with no EPSDT visits. EPSDT visits do not affect the likelihood of NED visits among NHW children as indicated in the far right hand columns of Table 3.

\section{Non - Linear Cliff Model Results}

The estimates from the 'cliff' specification are virtually identical to those of the random effects model but fewer of the estimates are statistically significant because almost one-half of the samples is omitted. Medicaid insurance still reduces the likelihood of NED visits, while gaps in insurance coverage more than doubles the likelihood an NED visit. The likelihood of NED visits for Hispanic children with 7 or more EPSDT is approximately 2 percentage points in each and every year after two years of age, or about a 30 percent reduction in NED visits (Table 4).

\begin{tabular}{|c|c|c|c|c|}
\hline & \multicolumn{2}{|l|}{ Hispanics } & \multicolumn{2}{|c|}{ Non - Hispanic NHWs } \\
\hline & coefficient & prob. signif. & coefficient & prob. signif. \\
\hline Intercept & -2.87 & $<0.001$ & -3.17 & $<0.001$ \\
\hline EPSDT lots of visits dummy & 0.10 & 0.476 & -0.18 & 0.459 \\
\hline after (EPSDT lots of visits) & -0.44 & 0.024 & 0.19 & 0.596 \\
\hline after & 0.54 & 0.001 & 0.48 & 0.094 \\
\hline male & 0.20 & 0.033 & -0.17 & 0.320 \\
\hline health condition & -0.10 & 0.474 & -0.29 & 0.317 \\
\hline Medicaid insurance & -0.23 & 0.279 & -0.52 & 0.029 \\
\hline Private insurance coverage & 0.21 & 0.292 & 0.26 & 0.258 \\
\hline gaps in insurance coverage & 1.33 & $<0.001$ & 1.49 & $<0.001$ \\
\hline Diff. - in - diff. marginal effect & \multirow{2}{*}{\multicolumn{2}{|c|}{$\begin{array}{l}-0.0180 \\
(0.13)\end{array}$}} & \multirow{2}{*}{\multicolumn{2}{|c|}{$\begin{array}{l}0.0196 \\
(0.14)\end{array}$}} \\
\hline (probability significance) & & & & \\
\hline Log likelihood & \multicolumn{2}{|l|}{-1685.9623} & \multicolumn{2}{|l|}{-518.7077} \\
\hline Sample size (children years) & \multicolumn{2}{|l|}{5990} & \multicolumn{2}{|l|}{2166} \\
\hline Test: insurance effects & \multicolumn{2}{|l|}{$<0.001$} & \multicolumn{2}{|l|}{0.001} \\
\hline Test year fixed effects & \multicolumn{2}{|l|}{$<0.001$} & \multicolumn{2}{|l|}{$<0.001$} \\
\hline Test: zipcode effects & \multicolumn{2}{|l|}{0.012} & \multicolumn{2}{|l|}{0.523} \\
\hline Test: Hispanic / NHW equality & \multicolumn{4}{|l|}{0.712} \\
\hline
\end{tabular}

Table 4: NED emergency department visits per child year logistic regressions, comparing most visit responses $(>=7)$ with least visit responses $(<=1)$ by age 2 . All probability significance levels are adjusted for clustering at the individual level, and adjusted for heteroskedasticity using Robust Standard Errors. The difference - in - difference marginal effect in the last line is calculated using the Ai and Norton [15] corrections. Year fixed effects and Zip Code fixed effects are included in these models, but not reported in the tables. When we constrain hispanics and non hispanic children to have the same coefficient values, the resulting "after ${ }^{\star} E P S D T "$ coefficient is -0.3023 , significant at the $8 \%$ level. 
Citation: Butler RJ, Johnson WG (2016) EPSDT Care and Emergency Department Visits for Non-Urgent Care: Hispanic vs. Non-Hispanic Children in a Border Community. Health Econ Outcome Res Open Access 2: 112. DOI: 10.4172/2471-268X.1000112

\section{Non - Parametric Age Model Results}

This generalization leaves most of the results unchanged but increases the estimated effect of EPSDT service on NED visits. The estimated difference - in - difference effect, for example, falls from -0.0516 in Table 3 to -0.0644 in Table 5, implying that EPSDT visits decrease NED visits by 0.5 percentage points, or a $3.5\left(-0.5^{*} 7\right)$ percentage point decrease for a receiving the recommended number of visits by age two. This is a 50 percent reduction, each year, in the likelihood of using an ED for NED (Table 5).

\begin{tabular}{|c|c|c|c|c|}
\hline & \multicolumn{2}{|l|}{ Hispanics } & \multicolumn{2}{|c|}{ Non - Hispanic NHWs } \\
\hline & coefficient & prob. signif. & coefficient & prob. signif. \\
\hline Intercept & -2.55 & $<0.001$ & -1.81 & 0.031 \\
\hline EPSDT visits (by 2nd year) & 0.03 & 0.156 & -0.01 & 0.690 \\
\hline after EPSDT visits & -0.06 & 0.008 & 0.01 & 0.743 \\
\hline age $=0$ & 0.12 & 0.160 & -0.22 & 0.182 \\
\hline age $=1$ & -0.07 & 0.584 & -0.44 & 0.135 \\
\hline age $=2$ & -0.21 & 0.292 & -0.62 & 0.006 \\
\hline age $=3$ & 0.31 & 0.086 & 0.02 & 0.915 \\
\hline age $=4$ & 1.13 & $<0.001$ & 1.22 & 0.000 \\
\hline age $=5$ & -0.21 & 0.697 & -2.21 & 0.017 \\
\hline age $=6$ & 0.47 & 0.394 & -1.14 & 0.217 \\
\hline age $=7$ & 0.52 & 0.329 & -1.24 & 0.155 \\
\hline age $=8$ & 0.36 & 0.499 & -1.56 & 0.082 \\
\hline age $=9$ & 0.58 & 0.293 & -1.71 & 0.066 \\
\hline male & -0.98 & 0.079 & -0.81 & 0.256 \\
\hline health condition present & 0.42 & 0.410 & -1.74 & 0.051 \\
\hline Medicaid insurance & 0.68 & 0.220 & -2.00 & 0.095 \\
\hline Private insurance coverage & 0.19 & 0.752 & -0.97 & 0.297 \\
\hline gaps in insurance coverage & 0.34 & 0.574 & -0.05 & 0.954 \\
\hline Log likelihood & \multicolumn{2}{|l|}{-1964.5721} & \multicolumn{2}{|l|}{-544.9320} \\
\hline Sample size (children years) & \multicolumn{2}{|l|}{6995} & \multicolumn{2}{|l|}{2323} \\
\hline Test: insurance effects & \multicolumn{2}{|l|}{$<0.001$} & \multicolumn{2}{|l|}{$<0.001$} \\
\hline Test year fixed effects & \multicolumn{2}{|l|}{$<0.001$} & \multicolumn{2}{|l|}{$<0.001$} \\
\hline Test: zipcode effects & \multicolumn{2}{|l|}{0.015} & \multicolumn{2}{|l|}{0.191} \\
\hline Test: Hispanic / NHW equality & \multicolumn{4}{|c|}{$53.83(0.016)$} \\
\hline
\end{tabular}

Table 5: NED visits per child year logistic regressions. All probability significance levels are adjusted for clustering at the individual level, and adjusted for heteroskedasticity using Robust Standard Errors. The combined sample interaction effects was -0.0419 with probability significance of 0.031. (We do not present the Ai and Norton [15] results with individual age dummies as this correction is not readily calculated for those specifications.) Year fixed effects and Zip Code fixed effects are included in these models, but not reported in the table.

\section{Discussion}

We discussed, in a previous section, many studies showing that Hispanic children experience lower health status than Non - Hispanic white children and are less likely to receive regular primary care. These facts are likely to be one of the reasons that our results consistently show that the payoff to EPSDT services is higher for Hispanic children than their Non - Hispanic counterparts. That is, that the return to 
Citation: Butler RJ, Johnson WG (2016) EPSDT Care and Emergency Department Visits for Non-Urgent Care: Hispanic vs. Non-Hispanic Children in a Border Community. Health Econ Outcome Res Open Access 2: 112. DOI: 10.4172/2471-268X.1000112

Page 7 of 7

investments in screening and preventive care has a greater marginal benefit because the potential savings are higher. In our 'best' model (least parametric restrictions) receipt of the recommended number of EPSDT visits by age two reduces the use of the ED for NED care in each subsequent year by fifty percent.

Our results are limited to only one of the possible benefits of EPSDT services, namely the avoidance of ED visits for NED over a period of approximately five years. It is reasonable to presume that both Hispanic and non-Hispanic children receive other benefits and that the benefits recur for longer periods of time.

It is important to recognize that our estimates of the benefits of EPSDT visits are in addition to the positive effects of health insurance coverage. Thus, increases in access to EPSDT services associated with health insurance coverage are not included in the benefits of EPSDT services that we measure. Health insurance coverage, not surprisingly, is an extremely important influence on NED visits Children who were uninsured for all or part of a given year were twice as likely to use the ED for NED care during the year. AHCCCS / SCHIP coverage is especially important in our sample, partly because such a large portion of the children were covered by AHCCS or SCHIP. An important aspect of AHCCCS coverage during this period was the assignment of patients to a primary care provider, typically a paediatrician, guaranteeing children on going source of regular care [16].

The costs and benefits of EPSDT services were not the subject of this article but one can speculate about that using a very approximate comparison. The mean payment (not charge, but payment) for an ED visit in 2004 in Arizona was \$795.57, while an average ambulatory visit payment was $\$ 162.64$ [17]. Hence, the total cost of seven EPSDT visits was $\left(7^{*} \$ 162.64\right)$, or $\$ 1,138.48$. The avoidance of 2 NED visits would more than offset the costs of the EPSDT visits, independent for any other benefits the EPSDT visits would have on children's health and wellbeing. A more rigorous analysis of the benefits and costs would be a useful topic for future research.

Finally, while it the relatively large number of $\mathrm{H}$ children in the sample allow for more efficient estimated effects than many prior studies, the relatively large fraction of $\mathrm{H}$ children in this border population may be interacting with the smaller NHW population in ways that are atypical of other $\mathrm{H} / \mathrm{NHW}$ samples, possibly leading to external validity of these results relative to other $\mathrm{H} / \mathrm{NHW}$ samples.

\section{Conclusion}

The role of EPSDT services is an important consideration in the potential effectiveness of the innovations created as part of the Affordable Care Act (ACA). The ACA mandates Medicaid coverage to children in households with incomes up to $138 \%$ of the Federal Poverty Level. All uninsured children not previously on Medicaid will be eligible for EPSDT benefits under the ACA.

Our results suggest that the ACA can have two important impacts on Hispanic children. The ACA can directly reduce NED visits to the extent that it encourages more frequent use of EPSDT visits and it should reduce gaps in insurance coverage.

This study offers the advantage of a longitudinal study that captures at least one of the benefits of EPSDT services and the effects of health insurance coverage. The sample size is quite large and includes a very large number of Hispanic children, a growing segment of the U.S. population. Inferences, however, must be made subject to the limitation that the locale is a border community and that the Hispanic children are overwhelmingly Mexican in origin while Hispanics in other states include persons from central and South America.

\section{Acknowledgement}

William Johnson oversaw the data collection. Richard Butler did the data analysis. Both authors equally contributed to the writing of the research and had full access to all the data in the study and take responsibility for the integrity of the data and the accuracy of the data analysis. The authors thank Gevork Harootunian for help with data collection. The authors acknowledge the research support of the Center for Health Information Research at Arizona State University. There are no conflicts of interest in this research, including financial interests, affiliations, or relationships.

\section{References}

1. Cunningham PJ (2006) Medicaid / SCHIP cuts and hospital emergency department use. Health Aff (Millwood) 25: 237-247.

2. Selden TM, Hudson JL (2005) How much can really be saved by rolling back SCHIP? The net cost of public health insurance. Inquiry 42: 16 -28.

3. American Academy of Pediatrics (2000) Recommendations for Preventive Pediatric Health Care Pediatrics 105: 645-646.

4. http://www.aap.org/family/tippmain.htm.

5. http://brightfutures.aap.org/pdfs/AAP\%20Bright\%20Futures \%20Periodicity\%20Sched\%20101107.pdf

6. Hakim RB, Ronsaville DS (2002) Effect of compliance with health supervision guidelines among US infants on emergency department visits. Arch Pediatr Adolesc Med 156: 1015-1020.

7. Flores G, Olson L, Tomany Korman SC (2005) Racial and ethnic disparities in early childhood health and health care. Pediatrics 115: e183e193.

8. Lara M, Akinbami L, Flores G, Morgenstern H (2006) Heterogeneity of childhood asthma among Hispanic children: Puerto Rican children bear a disproportionate burden. Pediatrics 117: 43-53.

9. Dick AW, Brach C, Allison RA, Elizabeth Shenkman E, Shone LP, et al. (2004) SCHIP's Impact in Three Vulnerable States: How Do the Most Vulnerable Children Fare. Health Aff (Millwood) 23: 63-74.

10. Chung PJ, Schuster MA (2004) Access and Quality in Child Health Services: Voltage Drops. Health Aff (Millwood) 23: 77-88.

11. Ronsaville DS, Hakim RB (2000) Well child care in the United States: racial differences in compliance with guidelines. Am J Public Health 90: 1436-1443.

12. http://oig.hhs.gov/oei/reports/oei-05-13-00690.pdf.

13. Luo X, Liu G, Frush K, Hey LA (2003) Children's health insurance status and emergency department utilization in the United States. Pediatrics 112: 314-319.

14. Sarver JH, Cydulka RK, Baker DW (2002) Usual source of care and nonurgent emergency department use. Acad Emerg Med 9: 916-923.

15. Ai C, Norton EC (2003) Interaction Terms in Logit and Probit Models. Economic Letters 80: 123-129.

16. Johnson WG, Rimsza ME (2004) The effects of access to pediatric care and insurance coverage on emergency department utilization. Pediatrics 113: 483-487.

17. Rimsza M, Butler RJ, Johnson WG (2007) Impact of Medicaid Disenrollment on Health Care Use and Cost. Pediatrics 119: 1026-1032. 